\title{
COMMENTARY
}

\section{Fish oil-containing lipid emulsions in patients with sepsis}

\author{
Konstantin Mayer* and Werner Seeger \\ See related research by Barbosa et al., http://ccforum.com/content/14/1/R5
}

\begin{abstract}
Lipid emulsions based on soybean oil have been an integral part of parenteral nutrition supplying $n-6$ fatty acids, with possible negative effects in critically ill patients. Newer lipid emulsions supply less n-6 fatty acids. In addition, fish oil-based lipids may be included in the lipid component of parenteral nutrition. While clinical benefits of lipid emulsions with a reduced fraction in n-6 lipids and the addition of fish oil have been described in postoperative patients, data are less clear in critically ill or septic patients. Recent data suggest that beneficial effects may be achieved when used early but clearly more data are needed to come to a definitive conclusion. The present commentary will highlight current data in critically ill and septic patients and the use of fish oil as a part of parenteral nutrition.
\end{abstract}

Parenteral nutrition is often used in critically ill patients when enteral nutrition is not sufficient or is not fully installed. Lipid emulsions are an integral part of parenteral nutrition as they offer a high caloric density and prevent the fatty liver degeneration of long-term carbohydrate-based parenteral nutrition. Beyond use as a caloric source, lipids may influence the immune response. The standard lipid emulsion is based on soy bean oil (SO) supplying $\mathrm{n}-6$ polyunsaturated fatty acids thought necessary to prevent essential fatty acid deficiency. In critically ill patients and in animal models, however, a negative impact of these lipid emulsions has been described. Barbosa and colleagues now report the effects of a new lipid emulsion on clinical and experimental parameters in patients with systemic inflammatory response syndrome and sepsis [1]. How do these effects integrate into our current concepts?

*Correspondence: Konstantin.Mayer@uglc.de

University of Giessen Lung Center, Medical Clinic II, Justus-Liebig-University Giessen, Klinikstraße 36, D-35392 Giessen, Germany
Use of SO-based lipid emulsions increases availability of free arachidonic acid, the precursor fatty acid of lipid mediators in septic patients [2]. A deterioration of the $\mathrm{PaO}_{2} / \mathrm{FiO}_{2}$ ratio has been attributed to fast infusion (6 hours) instead of slow infusion (24 hours) of a SObased lipid emulsion in patients with acute respiratory distress syndrome due to generation of arachidonic acidderived prostaglandins and thromboxane [3]. In a simplified model, sepsis may be described as starting with a hyperinflammatory phase followed by a phase of immune suppression. When used early in septic patients, SO-based lipid emulsions have been shown to increase $e x$ vivo cytokine generation in a small study [2]. These lipid emulsions also led to an increased apoptosis of splenic lymphocytes in a murine model, amplifying this key feature of immune suppression in late sepsis [4]. Recent European Society for Parenteral and Enteral Nutrition guidelines recommend using other available lipid emulsions with a reduced content of n- 6 polyunsaturated fatty acids instead of SO-based lipid emulsions [5].

Addition of a pure fish oil (FO)-based emulsion to a non-FO-containing emulsion or use of FO-containing lipid emulsions may offer alternative options to provide lipids. With this approach a supply of $\mathrm{n}-3$ fatty acids may be combined with a reduced deliverance of $n-6$ lipids. Interestingly, the recently discovered n-3 fatty acidderived resolvins and protectins are key mediators for the active resolution of inflammatory processes and their application has shown improved outcome in a murine model of abdominal sepsis [6]. Results from smaller trials and multicenter trials including FO in the parenteral nutrition in patients after major surgery herald a good clinical benefit, including a reduced length of stay (for example, [7]).

A larger single-centre trial in 166 critically ill patients comparing a lipid emulsion based on SO and mediumchain triglycerides (LCT/MCT) versus the same emulsion supplemented with FO, however, failed to detect a clinical benefit [8]. This study was planned to detect a faster reduction in biomarkers modeled after the time course in postoperative patients; however, the authors failed to prove a significant reduction in both 
groups. A possible explanation may be that they started to use the lipid emulsions after the initial inflammatory process was already terminated. All secondary outcome parameters - such as, for example, infectious rate, length of stay, and mortality - were not significantly different.

This study is contrasted by the recent trial of Barbosa and coworkers [1]. They randomized 25 patients with systemic inflammatory response syndrome and sepsis to receive parenteral nutrition with a $\mathrm{LCT} / \mathrm{MCT}$ emulsion or a LCT/MCT/FO lipid emulsion supplied continuously over 5 days, demonstrating a highly significant faster reduction in IL-6 in plasma in the LCT/MCT/FO group. In contrast to a previous study by Friesecke and colleagues [8], the patients had a higher mortality rate and were included earlier, exhibiting high IL-6 plasma concentrations at study entry. Barbosa and colleagues could also show a significantly faster improvement of the oxygenation parameter, as determined by the $\mathrm{PaO}_{2} / \mathrm{FiO}_{2}$ ratio in the LCT/MCT/FO group. While other clinical outcome parameters were not different between the two groups, a trend for a shorter length of stay in hospital was found.

Keeping in mind the small number of patients included, the interesting data of the study are supported by another small trial in 40 critically ill patients with severe acute pancreatitis [9]. In a double-blind randomized study, 5 days of parenteral nutrition using a SO-based lipid emulsion was compared with SO $(80 \%$ of the lipid component) with additional FO early in the course of the disease. The authors reported a significant reduced need for renal replacement therapy and a faster improvement in the $\mathrm{PaO}_{2} / \mathrm{FiO}_{2}$ ratio in the $\mathrm{SO} / \mathrm{FO}$ group. Furthermore, improved liver function was reported in critically ill patients when including a fraction of FO in the parenteral nutrition [10], and parenteral nutrition-associated liver disease was reversed in a cohort of children receiving long-term parenteral nutrition when exchanging a SObased lipid emulsion for a FO-based lipid emulsion [11].

What may we conclude from the available data? It seems prudent to use lipid emulsions with a reduced fraction of n-6 polyunsaturated fatty acids as recommended by the European Society for Parenteral and Enteral Nutrition [5]. The effect of FO seems best when used early in critically ill patients. Most studies used between 0.1 and $0.2 \mathrm{~g} / \mathrm{kg} /$ day FO as part of the parenteral nutrition. This assumption is supported by the analysis of a database in 661 critically ill patients, including patients with sepsis, where best outcome data were found in this dose range [12]. The promising data from Barbosa and colleagues in septic patients should lead to trials examining the effects of FO in larger cohorts of patients.

\section{Abbreviations}

FO, fish oil; IL, interleukin; LCT, long-chain triglycerides; MCT, medium-chain triglycerides; $\mathrm{PaO}_{2} / \mathrm{FiO}_{2^{\prime}}$, partial pressure of arterial oxygen/fraction of inspired oxygen; SO, soy bean oil.

\section{Acknowledgements}

Supported by the Excellence Cluster Cardio-Pulmonary System funded by the Deutsche Forschungsgemeinschaft and the Clinical Research Unit Pneumonia funded by the Federal Ministry of Education and Research (BMBF).

\section{Competing interests}

KM has received speaking fees from Abbott, Baxter, BBraun, Fresenius Kabi, and Nestle. WS declares that they have no competing interests.

Published: 10 March 2010

\section{References}

1. Barbosa VM, Miles EA, Calhau C, Lafuente E, Calder PC: Effects of a fish oil containing lipid emulsion on plasma phospholipid fatty acids, inflammatory markers, and clinical outcomes in septic patients: a randomized, controlled clinical trial. Crit Care, 14:R5.

2. Mayer K, Gokorsch S, Fegbeutel C, Hattar K, Rosseau S, Walmrath D, Seeger W, Grimminger F: Parenteral nutrition with fish oil modulates cytokine response in patients with sepsis. Am J Respir Crit Care Med 2003, 167:1321-1328.

3. Suchner U, Katz DP, Furst P, Beck K, Felbinger TW, Senftleben U, Thiel M, Goetz $A E$, Peter $K$ : Effects of intravenous fat emulsions on lung function in patients with acute respiratory distress syndrome or sepsis. Crit Care Med 2001, 29:1569-1574.

4. Bi MH, Ott J, Fischer T, Hecker M, Dietrich H, Schaefer MB, Markart P, Wang BE, Seeger W, Mayer K: Induction of lymphocyte apoptosis in a murine model of acute lung injury - modulation by lipid emulsions. Shock 2010, 33:179-188.

5. Singer P, Berger MM, Van den Berghe G, Biolo G, Calder P, Forbes A, Griffiths R, Kreyman G, Leverve X, Pichard C: ESPEN guidelines on parenteral nutrition: intensive care. Clin Nutr 2009, 28:387-400.

6. Spite M, Norling LV, Summers L, Yang R, Cooper D, Petasis NA, Flower RJ, Perretti M, Serhan CN: Resolvin D2 is a potent regulator of leukocytes and controls microbial sepsis. Nature 2009, 461:1287-1291.

7. Wichmann MW, Thul P, Czarnetzki HD, Morlion BJ, Kemen M, Jauch KW: Evaluation of clinical safety and beneficial effects of a fish oil containing lipid emulsion (Lipoplus, MLF541): data from a prospective, randomized, multicenter trial. Crit Care Med 2007, 35:700-706.

8. Friesecke S, Lotze C, Kohler J, Heinrich A, Felix SB, Abel P: Fish oil supplementation in the parenteral nutrition of critically ill medical patients: a randomised controlled trial. Intensive Care Med 2008, 34:1411-1420.

9. Wang X, Li W, Li N, Li J: Omega-3 fatty acids-supplemented parenteral nutrition decreases hyperinflammatory response and attenuates systemic disease sequelae in severe acute pancreatitis: a randomized and controlled study. J Parenter Enteral Nutr 2008, 32:236-241.

10. Antebi H, Mansoor O, Ferrier C, Tetegan M, Morvan C, Rangaraj J, Alcindor LG: Liver function and plasma antioxidant status in intensive care unit patients requiring total parenteral nutrition: comparison of 2 fat emulsions. J Parenter Enteral Nutr 2004, 28:142-148.

11. de Meijer VE, Gura KM, Le HD, Meisel JA, Puder M: Fish oil-based lipid emulsions prevent and reverse parenteral nutrition-associated liver disease: the Boston experience. J Parenter Enteral Nutr 2009, 33:541-547.

12. Heller AR, Rossler S, Litz RJ, Stehr SN, Heller SC, Koch R, Koch T: Omega-3 fatty acids improve the diagnosis-related clinical outcome. Crit Care Med 2006, 34:972-979.

doi:10.1186/cc8882

Cite this article as: Mayer K, Seeger W: Fish oil-containing lipid emulsions in patients with sepsis. Critical Care 2010, 14:128. 